\title{
CONTRIBUIÇÃO EDUCATIVA E TERAPÊUTICA DA HIDROTERAPIA NA QUALLIDADE DE VIDA DE PACIENTES PORTADORES DE OSTEOPOROSE: ANÁLISE DE UM PROGRAMA DE EDUCAÇÃO PARA A SAÚDE ${ }^{1}$
}

\section{CONTRIBUTION EDUCATIONAL AND THERAPY OF HYDROTHERAPY IN QUALITY OF LIFE OF PATIENTS WITH OSTEOPOROSIS: REVIE ${ }^{1} \mathrm{~W}_{\text {OF }}$ A PROGRAM OF EDUCATION FOR HEALTH}

\author{
Marcos Antonio de Farias \\ Programa Stricto Sensu do Centro Universitário Plínio Leite
}

\section{Resumo}

Este trabalho implementou e analisou um Programa de Educação para a Saúde voltado a portadores de osteoporose. A osteoporose constitui grave problema de saúde pública, sobretudo pelas fraturas. No Brasil $30 \%$ das mulheres que passaram pela menopausa irão desenvolver a osteoporose (GOMES e GARCIA, 2006). A metodologia foi participativa e teve como cenário uma Clinica de Fisioterapia. O universo foi composto por um fisioterapeuta e uma nutricionista, e por três voluntárias do sexo feminino, entre 50 e 70 anos. Contou com a análise clínica dos integrantes, palestras educativas e ações de hidroterapia. Confirmou a importância da terapia em grupo, de uma equipe multidisciplinar e a necessidade de palestras educativas no tratamento da osteoporose. Tais resultados oportunizaram a construção de um novo Programa de Educação para a Saúde voltada para pacientes osteoporóticos e traz como novidade, uma proposta de interface do Ensino e Saúde com a área de Ensino de Ciências.

Palavras-chave: Osteoporose, Hidroterapia, Qualidade de Vida, Espaço não-formal de Ensino, Ensino de Ciências.

\begin{abstract}
This study aimed to examine and implement a program of health education for osteoporosis'. Osteoporosis is a serious public health problem, with major social impact on its considerable morbidity and high social cost, especially for fractures. In Brazil $30 \%$ of the women who have gone through menopause will develop osteoporosis, with and overall methodology applied was participatory, taking place in a Physical Therapy Clinic in the around five million women (GOMES AND GARCIA, 2006). That way, the city of Cabo Frio, Rio de Janeiro, Brazil. The environment was a compound of professionals involved in the research, a physical therapist and a nutritionist, and three female volunteers, around their 50's and 70's. The intervention assures the importance of group therapy and multidisciplinary team in the osteoporosis treatment. These results

1 Este trabalho é oriundo da Dissertação "CONTRIBUIÇÃO EDUCATIVA E TERAPÊUTICA DA HIDROTERAPIA NA QUALIDADE DE VIDA DE PACIENTES PORTADORES DE OSTEOPOROSE: ANÁLISE DE UM PROGRAMA DE EDUCAÇÃO PARA SAÚDE”, defendida em 2008, no Curso de Mestrado Profissional em Ensino de Ciências da Saúde e do Ambiente/UNIPLI.
\end{abstract}


created an opportunity for a new Health Education Program specific for osteoporosis. Suggesting a work between Education/Health and Science Education.

Key words: Osteoporosis, Hydrotherapy, Life Quality, Area Non-Regular Education Place, Science Education

\section{Introdução}

Segundo Carvalho e outros (2004) a osteoporose é uma doença sistêmica que resulta em reduzida massa óssea e deterioração da micro-arquitetura do tecido ósseo, levando à fragilidade mecânica e consequente predisposição à fraturas com trauma mínimo, atingindo a todos, em especial, mulheres após a menopausa. A doença é considerada uma importante questão de saúde pública mundial devido a sua alta prevalência, em função dos seus efeitos devastadores na saúde física e psicossocial, com grandes prejuízos financeiros. Esta patologia causa invalidez pelas deformidades e incapacidades dos indivíduos afetados e, pelo demorado tratamento das fraturas decorrentes da enfermidade, gera um ônus elevado. A etiologia da perda de massa óssea é complexa, multifatorial, inclui hereditariedade, etnia, idade avançada, baixo peso corporal, deficiência hormonal, excessivo consumo de álcool, inatividade física, tabagismo e fatores nutricionais.

Embora já estejam bem estabelecidos, os benefícios nas mudanças dos hábitos de vida como um importante fator modificável relacionado à saúde óssea, a sua importância e o conhecimento de que a prevenção da perda de massa óssea pode ser feita com alimentação balanceada e a prática regular de exercício físico nem sempre é do conhecimento da população.

Para a promoção de saúde, educar é contribuir para a autonomia das pessoas, é considerar a afetividade, a capacidade criadora e a busca da felicidade como indissociáveis das demais dimensões da vida humana. Promover a saúde não pode ser uma ação deslocada dos sentidos atribuídos à vida que se somam aos saberes acumulados, tanto pelas ciências, quanto pelas tradições culturais locais e universais. Alguns programas educativos têm surgido no sentido de orientar os participantes em nível de prevenção e tratamento da osteoporose (CARVALHO E OUTROS, 2004).

Diante desse quadro e das informações de que a população brasileira está envelhecendo, são importantes, não apenas ações de ensino visando à prevenção da osteoporose, como também práticas educativas que possam tornar a vida dessa população mais próxima das condições de qualidade de vida. 
A hidroterapia como recurso educativo e terapêutico, utilizado no tratamento da osteoporose, tem um papel muito importante e pode estar presente durante quase todas as etapas do tratamento. A sua indicação está relacionada com o conhecimento e compreensão das suas propriedades, dos seus princípios físicos e mecânicos e também do tipo de lesão que está sendo tratada (RODRIGUES, 1993).

Segundo Campion (2000), o uso da hidroterapia no tratamento de doenças ortopédicas e reumáticas é amplamente reconhecido. Isso se deve pela possibilidade da água ser aquecida e envolver o corpo do paciente, resultando na diminuição da dor e do espasmo muscular, como também, pela flutuabilidade do corpo humano na água, o que alivia o estresse em todas as articulações, especialmente nas articulações sustentadoras do peso.

Em se tratando especificamente do paciente portador de osteoporose entendemos que esse é um paciente especial e deve ser tratado como tal, isto é, com muitos cuidados e sempre nestes casos a hidroterapia será sempre bem indicada. $\mathrm{O}$ paciente deve ser encorajado a realizar todo o trabalho educativo e terapêutico de fortalecimento dentro da piscina, o que diminui o risco de fratura eminente, que acompanha os pacientes osteoporóticos (TISNSLEY apud CAMPION, 2000).

A hidroterapia vem sendo cada vez mais estudada pelos profissionais de saúde e áreas afins. Esta técnica visa utilizar os efeitos fisiológicos da água aquecida aproximadamente de 31 a 33 graus Celsius no inverno e 29 para o verão (SOARES, 1999).

De acordo com Campion (2000) o tratamento pode ser aplicado em grupo ou individualmente. As terapias em grupo têm apresentado vantagens consideráveis. Muitos dos benefícios são semelhantes para todos, porém existem alguns diferentes, de acordo com a história e patologia de cada indivíduo. A motivação e a socialização das pessoas estão entre as vantagens alcançadas com o tratamento em grupo realizado na água.

A hidroterapia integra muitas técnicas de tratamento. Movimentos funcionais são enfatizados usando padrões sinérgicos, estabilização, correção postural e biomecânica articular. A flexibilidade desse tipo de programa é limitada somente pela criatividade do terapeuta. Essa flexibilidade permite a incorporação de numerosos tipos de técnicas e exercícios usados em combinação e adaptadas ao paciente individualmente (MCNEAL apud RUOT E OUTROS, 2000). 
Esta pesquisa objetivou, portanto, a implementação e a análise de um Programa de Educação em Saúde, cuja ênfase foi o tratamento da osteoporose por meio da hidroterapia vinculada a outras práticas em saúde, visando melhorar a qualidade de vida de portadores de osteoporose por meio de uma interface entre a hidroterapia e a educação para a saúde.

Defini-se o conceito de qualidade de vida, meta a ser alcançada no Programa implementado e analisado, como sendo o bem-estar pessoal, posse de bens materiais, acesso à educação, lazer e meios de saúde; e participação em ações coletivas. No caso específico do Programa, serão avaliadas as condições de bem-estar pessoal.

\section{Material e Métodos}

O conjunto de sujeitos participantes da pesquisa foi composto por três mulheres, todas moradoras do município de Cabo Frio com idades que variaram de 61 a 79 anos com média de idade de 71 anos. Como critério de inclusão, as voluntárias apresentaram exame de densitometria óssea, com laudo assinado pelo médico responsável confirmando diagnóstico de osteoporose. A pesquisa se realizou na Fisioterapia Clínica São Vicente, situada na cidade de Cabo Frio, município integrante da Região dos Lagos, Estado do Rio de Janeiro. Seguiu um modelo de estudo qualitativo, tipo participante, já que a proposta do projeto é ter o "observador como participante".

As pacientes foram recrutadas através de carta aberta, divulgada na própria clínica que serviu de palco para pesquisa, assim como nos comércios e nos serviços de saúde pública. Para uma análise da qualidade de vida antes e após o episódio da osteoporose, foi aplicado um questionário para avaliar o nível de satisfação pessoal, no relacionamento familiar e social dessas pacientes. Posteriormente, foram encaminhadas ao Setor de Nutrição para uma avaliação antropométrica, nutricional e alimentar, objetivando intervenção dietoterápica adequada. A partir do resultado das avaliações, a nutricionista orientou e prescreveu dieta alimentar individualizada de acordo com as necessidades nutricionais identificadas.

Após análise do exame de densitometria óssea assinado pelo médico responsável acusando positividade para osteoporose, o fisioterapeuta através da anamnese investigou sobre o inicio dos primeiros sintomas da doença, o estilo de vida dos sujeitos e a existência de outras patologias associadas. A partir daí, construiu a história da doença atual (HDA) e a queixa principal (QP), dados que nortearam a 
elaboração do protocolo de tratamento fisioterápico com base na cinesioterapia e hidroterapia.

Foi realizada aplicação de questionários abertos com as voluntárias selecionadas antes e depois da vivência do Programa de Educação para a Saúde, com a finalidade de verificar a representação dos mesmos sobre as suas condições de qualidade de vida e a influência do Programa citado na melhoria dessas condições. Foram analisadas através dos questionários as dificuldades e limitações impostas pela osteoporose, o conhecimento acerca da doença, as demandas dos pacientes diante do Programa implementado e as transformações por ele proporcionadas na qualidade de vida dos sujeitos participantes.

O Programa de Hidroterapia foi realizado as segundas, quartas e sextas feiras por um período de oito semanas, totalizando 24 sessões de 50 minutos cada.

A técnica hidroterápica utilizada no programa foi uma adaptação do método de Bad Ragaz que incorpora movimentos com padrão em planos anatômicos e diagonais, com resistência e estabilização fornecidas pelo próprio fisioterapeuta responsável. É uma técnica muito utilizada para portadores de patologias ortopédicas ou comprometimentos neurológicos (SKINNER E OUTROS,1998 apud CUNHA, 2001).

O programa implementado e analisado nessa pesquisa foi uma associação de técnicas ao já conhecido método de Bad Ragz dando surgimento a uma adaptação desse método: O VHC (Ventosa terapia- Hidrocinesioterapia- Crioterapia). Método globalizado onde as técnicas fisioterápicas acima citadas são aplicadas na hidroterapia, de acordo com a necessidade do paciente.

Cinesioterapia: terapêutica motora (através de movimentos) indicada para os indivíduos com uma deficiência geral ou particular (LAPIERRE, 1982).

Crio terapia: todo uso de gelo ou aplicações de frio para fins terapêuticos. Aplicação terapêutica de qualquer substância ao corpo que resulta em remoção do calor corporal, diminuindo assim a temperatura dos tecidos (KNIGTH, 2000).

Ventosa terapia: técnica da medicina tradicional chinesa que consiste na colocação de copos de ventosa no corpo sobre a pele para gerar sucção no local, provocando alivio da dor (CUNHA, 1996).

Para a realização dos exercícios aquáticos foram utilizados flutuadores e halteres. Em outros momentos os exercícios eram realizados utilizando a própria resistência da água. Os exercícios tinham como meta principal o ganho de massa óssea e muscular, melhora da força e resistência. 
As atividades aquáticas se iniciaram com caminhadas com duração de cinco minutos com objetivo de aquecer o organismo para os exercícios subseqüentes. Após o aquecimento, eram executados exercícios de extensão e flexão de membros inferiores. $\mathrm{Na}$ barra em decúbito dorsal, com ajuda de flutuadores, eram realizados exercícios de adução e abdução de membros inferiores e isometria de glúteos. Em decúbito ventral, ainda com flutuadores, foram exercitados os músculos extensores e flexores dos membros inferiores. Na posição de agachamento, imitando boxeadores e com alteres pesando $1 \mathrm{~kg}$, era exercitada a musculatura dos membros superiores, com movimentos de adução e abdução, flexão e extensão. Exercícios de propiocepção foram realizados tendo como recurso cama elástica subaquática.

Cada exercício era realizado com uma seqüência de 50 repetições, com intervalo de 3 minutos.

Para alívio de um processo doloroso ocorrido com duas das voluntarias durante algumas das sessões, foi utilizada a ventosa terapia durante dez minutos e posteriormente a crioterapia, durante vinte minutos. Essas técnicas complementares eram utilizadas com a paciente na borda da piscina. Vale ressaltar que após as primeiras três semanas de tratamento, as pacientes não apresentaram mais nenhum tipo de dor.

Embora a osteoporose, segundo Moreira e Xavier (2001), seja uma doença indolor, ela predispõe o organismo a outras patologias orteoarticulares dolorosas como osteoartrose, osteoartrite, etc. A ventosa terapia que, segundo Cunha (1996), é uma técnica que proporciona analgesia, é bem indicada e produz resultados bem satisfatórios para os pacientes que se queixam de dor.

A principal razão para o uso da crioterapia no paciente osteoporótico que apresenta dor como conseqüência das doenças associadas, se deve ao efeito analgésico e anestésico que esta técnica proporciona, permitindo que o exercício ativo aquático possa ser realizado de maneira indolor e de forma mais vigorosa. Embora ainda não exista uma resposta definitiva para o alivio ou redução da dor, algumas teorias explicam este efeito do frio pela liberação de endofirnas, pela inibição de neurônios espinhais e pela diminuição da transmissão nervosa da dor (KNIGHT, 2000).

$\mathrm{Na}$ verdade, o alivio da dor proporcionado pela associação destas técnicas à hidroerapia, é o que justifica o seu uso neste Programa que visa o aumento da densidade óssea, diminuição do risco de fraturas, retorno as atividades da vida diária, alivio da dor e conseqüentemente um aumento na qualidade de vida do paciente portador de osteoporose. 
O Programa também contou com palestra educativa realizada pelo fisioterapeuta, definindo a osteoporose, informando as formas de tratamento e prevenção da doença. Nesta ocasião, as pacientes interagiram com o fisioterapeuta, pois identificaram em sua fala, algumas das dificuldades por elas vivenciadas em função da osteoporose. Com a mesma proposta educativa, a nutricionista além das orientações nutricionais realizadas individualmente por ocasião da consulta, realizou palestra com objetivo de informar os alimentos ricos em cálcio e como elaborar uma alimentação rica neste nutriente, tão importante na prevenção e tratamento da osteoporose. A partir de um quadro com as quantidades de cálcio dos principais alimentos-fontes, foi elaborado verbalmente junto com o grupo um cardápio, contendo as quantidades de cálcio necessárias de acordo com a faixa etária e com o quadro de osteoporose apresentado pelas participantes. Esta atividade teve como objetivo a fixação do conteúdo exposto.

Ao final do programa foram informados ao grupo os resultados alcançados a partir da análise da densitometria ósseas realizada ao final do tratamento; avaliou-se a eficácia das técnicas fisioterápicas empregadas, pontuou-se junto às pacientes as melhoras alcançadas e avaliou-se o grau de satisfação alcançado com o programa.

\section{Resultados e Discussão}

O sujeito um, com 79 anos de idade, $1,57 \mathrm{~cm}$ de altura e $63,5 \mathrm{Kg}$ chegou ao Programa com osteoporose na coluna lombar (L1-L4) com T-Score de -2,7 e osteopenia no colo de fêmur com T-Score de - 0,7 . De acordo com as orientações da Organização Mundial de Saúde, os resultados obtidos são interpretados conforme a seguinte tabela:

1. Normal: T-Score maior ou igual a $-1,0$.

2. Osteopenia: T-Score entre $-1,0$ e $-2,5$.

3. Osteoporose: T-Score menor ou igual a $-2,5$.

O T-Score é o número de desvios padrões, acima ou abaixo do valor de referência de massa óssea de adultos jovens de mesmo sexo.

A paciente informou que, antes do desenvolvimento da osteoporose, fazia caminhadas diárias sem sentir dor. Depois de instalada a doença passou a ter dificuldades para realizar suas atividades diárias pelas dores causadas pela artrite nas mãos. Na avaliação da composição corporal, o Índice de Massa Corpórea (IMC) estava classificado como normal. Entretanto, na análise do inquérito alimentar para avaliação 
da qualidade da alimentação diária, constatou que a paciente ingeria poucos alimentos ricos em cálcio. No que diz respeito aos hábitos de vida, observou-se que a paciente era sedentária e que se expunha pouco ao sol, o que agravava ainda mais o quadro de osteoporose. Após o Programa, foi realizada uma nova densitometria óssea que constatou que na coluna lombar não havia mais osteoporose, pois o quadro havia regredido para osteopenia. Além disso, no colo do fêmur, onde se apresentava osteopenia, a conclusão diagnóstica final foi de normalidade. Os dados desta segunda densitometria óssea mostram na coluna lombar, na mesma região analisada anteriormente um T-Score de $-2,3$ e no colo do fêmur um T-Score de -0.6. Durante o tratamento, a paciente já relatava ausência de dores e melhora na sua capacidade de realizar movimentos, que anteriormente não conseguia realizar não somente por causa das dores, mas também, pela falta de mobilidade física.

Numa segunda consulta com a nutricionista, foi observado que novos hábitos alimentares haviam sido incorporados a sua alimentação diária, pois a paciente começou a ingerir uma quantidade bem maior de alimentos ricos em cálcio. Este comportamento foi resultado da palestra, dos questionamentos realizados em grupo e das trocas de experiências realizadas durante o tratamento.

Ao final do Programa, o sujeito um relatou grande satisfação com os resultados obtidos, pois as dores haviam desaparecido, o que a estava permitindo realizar tarefas anteriormente impossíveis de serem feitas, tais como "varrer casa, sair para ir a uma loja e caminhar".

O sujeito dois de 73 anos, $1,50 \mathrm{~cm}$ de altura e com 73,5 $\mathrm{Kg}$ entrou no Programa com osteoporose na lombar (L1-L4) com T-Score de -2,6 e no colo do fêmur, osteopenia com T-Score de -2,2. Na ocasião, a paciente relatou sentir fortes dores quando realizava suas atividades da vida diária, resultado das doenças que surgem como conseqüência da osteoporose. Nunca fez atividade física com regularidade, nem mesmo quando era jovem.

$\mathrm{Na}$ análise da composição corporal realizada pela nutricionista, ficou comprovado o diagnóstico de obesidade classe I, pois apresentava um Índice de Massa Corpórea (IMC) de 32,67. A Organização Mundial de Saúde (OMS) define sobrepeso quando o IMC encontra-se entre 25 e $29,9 \mathrm{Kg} / \mathrm{m} 2$ e obesidade, quando o IMC encontrase superior a $30 \mathrm{~kg} / \mathrm{m} 2$. A paciente apresentava também diagnóstico de diabetes mellitus. Na avaliação da ração alimentar, observou-se pouca ingestão de alimentos 
ricos em cálcio, o que compromete a saúde óssea e conseqüentemente a sua qualidade de vida.

Em decorrência do quadro clínico da paciente, a conduta dietoterápica adotada foi uma dieta hipocalórica, alimentos ricos em cálcio, controle na ingestão de carboidratos e isenção de açúcar. Tal conduta objetivou respectivamente, redução do peso, aumento nas taxas de cálcio e controle da glicemia. Conforme Claudino e Zanella (2005), em pacientes diabéticos, a redução de peso provoca melhora evidente do controle glicêmico.

No decorrer do tratamento, a paciente deixou claro que as dores haviam diminuído bastante.

Ao final do programa, após nova densitometria óssea encontrou-se o seguinte diagnóstico: coluna lombar (L1-L4) T-Score de -2,6 mantendo o diagnóstico de osteoporose. No colo do fêmur também manteve o diagnóstico de osteopenia, mostrando uma estabilidade do quadro. Por ser portadora de artrose em várias articulações em estágio já avançado, esta paciente ainda não conseguiu retornar a todas as suas atividades do cotidiano, mas já consegue andar com desenvoltura. Com isso, já se encontra mais independente dos seus familiares, sendo capaz de tomar banho sem ajuda, fazer sua comida e tomar o seu "banho de sol". Esta reconquista de ações significa, na verdade, a reconquista de valores perdidos em função da doença. Por isso, Minayo e outros (2000) afirmam que qualidade de vida está centralizada na capacidade de viver sem doenças ou de superar as dificuldades dos estados ou condições de morbidade.

O Sujeito três, com 61 anos, 1,68 de altura e 73,6 Kg chegou ao Programa com osteoporose na coluna lombar (L1-L4) com T-Score de -3,8 e no colo do fêmur com T-Score de -1,9. Antes do desenvolvimento da osteoporose relata não ter tantas dificuldades em seus afazeres domésticos, principalmente nas tarefas de cuidar de seu marido, um senhor diabético e com sérios problemas renais que o faz realizar hemodiálise semanalmente, sendo conduzido por ela ao local do tratamento. Após o surgimento da doença, em função das dores, os problemas começaram a surgir. Passou a andar com dificuldades e os trabalhos domésticos se tornaram mais estafantes e complexos, não só pelas dores no corpo, como também pela falta de mobilidade muscular. Em função desse impacto na vida dos indivíduos, é que Lerner e outros (2000) reconhecem a osteoporose como uma doença de relevante importância social e econômica, que gera invalidez e custos elevados. 
A avaliação antropométrica definiu seu IMC em $26,08 \mathrm{Kg} / \mathrm{m} 2$, o que a classifica com peso adequado para sua idade. Embora ingerisse alimentos fontes de cálcio, em função do quadro de osteoporose, as quantidades estavam aquém do necessário para garantir as necessidades ósseas. Daí a prescrição de alimentos ricos em cálcio para suprir as necessidades aumentadas deste mineral. A conclusão diagnóstica da densitometria óssea realizada ao final do programa, mostra que na coluna lombar na região de (L1-L4) manteve o quadro de osteoporose, com T-Score de -4,1 e no colo do fêmur também manteve quadro de osteopenia, com T-Score de -2,7. Pela análise dos exames, conclui-se que embora o diagnóstico tenha se mantido, houve ganho de massa óssea em uma das áreas analisadas.

Hoje consegue cuidar do seu marido com mais desenvoltura, pois não apresenta mais dores. Teve uma melhora considerável na postura e conseguiu realizar todos os exercícios propostos durante o tratamento. O resultado desse empenho se reflete no bom índice de melhora global alcançado. Atualmente chega à clínica caminhando, sem as queixas de cansaço que fazia no inicio do Programa. Percebe-se como esta nova condição melhorou a vida desta paciente, que viu suas expectativas alcançadas através do tratamento.

O grupo iniciou o Programa esperançoso e com grandes expectativas, pois trazia importantes dificuldades a serem vencidas. Inicialmente, ao avaliar o estilo de vida das voluntárias, observou-se que não realizavam atividade física regular, nem mesmo antes do surgimento da osteoporose.

Sabe-se que existe uma associação bem sólida entre a atividade física habitual, preservação da massa óssea e prevenção de osteoporose. A atividade física está positivamente relacionada com a DMO, sendo um importante fator na sua manutenção.

$\mathrm{Na}$ avaliação nutricional, foi percebida a ingestão de dieta alimentar pobre em alimentos ricos em cálcio e excesso de peso em uma das pacientes, o que agrava os riscos de dores e fraturas por gerar uma sobrecarga na estrutura óssea.

Ao final do programa, a partir da análise dos resultados encontrados, verificou-se a contribuição terapêutica que a hidrocinesioterapia trouxe na recuperação da massa óssea das pacientes osteoporóticas, sujeitos desta pesquisa. Através da densitometria final, pôde-se observar que uma paciente deixou de apresentar osteoporose em uma das áreas analisadas e as demais tiveram seu quadro estacionado, embora todas tenham conseguido um aumento na massa óssea. Acredita-se que com um tempo maior de tratamento, resultados melhores possam ser alcançados. 
Acredita-se que o sucesso deste programa se deve a princípio à multidisciplinaridade que permitiu que fossem contempladas todas as condutas necessárias para a recuperação dessas pacientes. $\mathrm{O}$ ajuste alimentar aliado à prática de exercícios físicos adequados, foi fundamental na melhora do estado clinico e conseqüentemente da qualidade de vida das voluntárias. Isso foi conseqüência da troca de informações e percepções dos profissionais envolvidos na problemática aqui exposta, o fisioterapeuta e a nutricionista.

Outro aspecto observado que contribuiu de forma relevante na conquista dos resultados aqui apresentados foi à integração que o tratamento em grupo proporcionou entre as pacientes. Havia uma cobrança saudável entre elas em relação à freqüência às sessões. Questionavam se estavam fazendo a alimentação de acordo com o que haviam aprendido na palestra e discutiam entre si as melhoras e mudanças alcançadas. Tornaram-se amigas e esse laço de amizade serviu de agente motivador para a assiduidade e pontualidade ao Programa. Percebeu-se que o comprometimento com o tratamento e com o alcance dos objetivos foi muito maior, do que se observa nos pacientes que realizam o seu tratamento individualmente, sem essa integração.

Uma observação importante, foi o fato de que ao ver que a colega conseguia realizar determinado exercício, gerava na outra uma motivação maior para se esforçar para realizar também apesar das suas dificuldades.

As palestras foram fundamentais para o esclarecimento e mudanças no estilo de vida dessas mulheres. Pois a partir daí, passaram a ver no banho de sol, na alimentação rica em cálcio e no exercício físico, recursos importantes não somente para a cura e controle da osteoporose, mas também como medidas preventivas a serem usadas por todos os indivíduos, principalmente as mulheres, onde a incidência de osteoporose é maior.

A pesquisa deixa clara a importância e eficácia da hidroterapia em grupo como um recurso fisioterápico no tratamento da osteoporose. Confirma a necessidade de incluir na prática profissional do fisioterapeuta, além do tratamento dentro da piscina, um horário destinado a parte educativa através de palestras, que esclareçam melhor os pacientes acerca de suas patologias e de como devem agir para um melhor alcance dos resultados esperados e assim obterem uma melhor qualidade de vida. Portanto, a equipe identifica e sugere para próximos estudos, um tempo maior destinado as palestras e oficinas em grupo, pois são momentos ricos em troca de informações e experiências tanto para os profissionais, quanto para os pacientes. 
Os resultados encontrados confirmam a importância da terapia em grupo e de uma equipe multidisciplinar que troque informações e interligue as ações, uma vez que a osteoporose depende de várias ações terapêuticas para que se obtenha um resultado satisfatório.

Conforme (TINSLEY apud CAMPION, 2000), as vantagens das atividades em grupo são encorajamento, estímulo, motivação e interação social, que ocorrem durante o tratamento com pessoas portadoras de deficiências semelhantes.

A eficácia deste tratamento se deu também pela associação de técnicas que proporcionou o alivio de alguns processos dolorosos secundários a osteoporose, trazidos pelas pacientes, que dificultaram em alguns momentos a realização de certos movimentos. Graças à técnica da crioterapia que segundo Knight (2000) causa analgesia e a ventosaterapia que conforme Pai (2005) exerce uma ação analgésica e de relaxamento muscular, essas dificuldades foram vencidas e o tratamento pôde ser realizado com sucesso.

\section{Conclusão}

Ao analisar os resultados desse Programa multidisciplinar para o tratamento de pacientes osteoporóticos, percebemos que algumas mudanças se fazem necessárias na prática hidroterápica usualmente utilizada, não só para o tratamento da patologia aqui estudada, mas também em todo o processo de reabilitação das demais doenças. Percebemos a importância das palestras educativas no comprometimento das pessoas, que entenderam seu papel como atores principais dentro do processo de sua própria recuperação. Conhecer a patologia, entender suas causas e como poderiam colaborar para antecipar a sua melhora e conseqüentemente melhorar as suas vidas, foi fundamental para que resultados satisfatórios pudessem ser alcançados.

Entretanto, ao analisar o tempo em que transcorreu o Programa com os resultados alcançados, conclui-se que um tempo maior de tratamento, traria resultados melhores dos que aqui foram encontrados. A partir daí, sugere-se a elaboração de outro Programa, com a participação de outros profissionais de saúde, e prevê ainda, uma interface com o Ensino de Ciências, já que o espaço do grupo será usado como espaço não-formal de ensino de ciências, com ênfase em conteúdos científicos que auxiliem o Ensino para a Saúde. 
Para tanto, é preciso ser muito mais do que um terapeuta na busca da cura ou da melhora das condições de saúde daquele que procura os serviços médicos na esperança de dias melhores. O profissional de saúde, que se preocupa com a qualidade da vida daquele que a ele se entrega em busca daquilo que representa um retorno dos seus valores perdidos, precisa além de cuidar e informar seu paciente, ser acima de tudo um educador.

O educador que não leve a sério sua formação, que não estude, que não se esforce para estar à altura de sua tarefa, não tem força moral para coordenar as atividades de sua classe, pois ensinar exige segurança, competência profissional e generosidade (FREIRE, 2004, p.92)

É com essa segurança, resultado da competência profissional, aliada a generosidade da qual nos fala Freire (2004), que nos remete à ética, que o profissional de saúde deve se revestir, para que possa sonhar com o bem estar de seu paciente, razão de ser de toda a sua formação e empenho.

Assim, estamos diante da necessidade de construir novas formas de relação, pautadas na solidariedade, ou seja, na escuta do outro, na valorização do saber popular em conjunto com o saber científico; saber popular significando o contexto no qual os sujeitos estão inseridos. Assim, precisamos estabelecer um novo operador ético, pautado na valorização do outro e isso no chamado Discurso Médico significa a valorização do saber do paciente acerca de sua vida e de seu próprio corpo.

\section{Referências}

1. CAMPION, M R. Hidroterapia - princípios e práticas. São Paulo: Manole, 2000. 2. CARVALHO, C; FONSECA, C; PEDROSA, J. I. "Educação para a saúde em osteoporose com idosos de um programa universitário: repercussões". Cad. Saúde Pública, Rio de Janeiro, 20(3): 719-726, maio e junho, 2004.

3. CLAUDINO, A.M; ZANELLA, M.T. Transtornos alimentares e obesidade. São Paulo: Manole, 2005.

4. CUNHA, A. A. Ventosaterapia. Sao Paulo: Ícone, 1996.

5. FREIRE, P. Pedagogia da autonomia. 29 edição. São Paulo: Paz e Terra, 2004.

6. GOMES, M. C. S. M. GARCIA, R. R. Comparação entre o tratamento no solo e na hidroterapia para pacientes com osteoporose: revisão da literatura. Revista Brasileira de Ciência da saúde, ano III, $\mathrm{n}^{\circ}$ 7, jan/mar, 2006.

7. KNIGTH, K. Crioterapia no tratamento das lesões esportivas. São Paulo: Manole, 2000.

8. LAPIERRE, A. A reeducação física. São Paulo: Manole, 1982.

9. LERNER, B R; LEI, D L M; CHAVES, S P; FREIRE, R D. O cálcio consumido por adolescentes de escolas publicas de Osasco, São Paulo. Revista de Nutrição, 
Campinas, v.13, n.1, jan./abr. 2000. Disponível em:

http://www.scielo.br/scielo.php?pid=s1415-

52732000000100008\&script=sci_arttext\&tIng=pt . Acessado em: 26 jul 2008.

10. R. Reabilitação aquática de pacientes com doenças reumáticas, Cap 11 In: In:

RUOTI R. G., MORRIS D. M. COLE A J. Reabilitação aquática, São Paulo: Manole, 2000.

11. MINAYO, M.C.S, HARTZ Z.M.A., BUSS, P. M. Qualidade de vida: um debate necessário - Ver. Ciência e Saúde Coletiva, volume 5, n 1. Rio de Janeiro, 2000. 12. MOREIRA A C., XAVIER A J. D., Doenças Osteometabólicas, Cap. 18, pp 327 328, Conceituação Epidemiológica, 2001, In: MOREIRA A C., CARVALHO M. A P. Reumatologia, diagnóstico e tratamento, $2^{\text {a }}$. Edição, Rio de Janeiro: Guanabara Koogan, 2001.

13. PAI, H.J. Acupuntura: de terapia alternativa a especialidade médica. São Paulo: CEIMEC, 2005.

14. RODRIGUES, A. Joelho no esporte: técnicas avançadas de avaliação e tratamento das lesões ligamentares. São Paulo: CEFESPAR, 1993.

15. SKINNER, A. T; THOMSON, A. M.. Exercises in Water, 3rd ed. London England, Baizziere Tindal, 1983. In: CUNHA, M. C. B., LABRONICE R. H. D. D., OLIVEIRA A S. B., GABBAI A A, Fisioterapia Brasil, Vol 2, n: 6, p. 329-392, Rio de Janeiro: Atlântica, Dezembro, 2001.

16. SOARES, M, P. Hidroterapia nas patologias de joelho - Rio de Janeiro: Sprint, 1999.

17. TINSLEY L. M, Doenças reumáticas - vantagens e desvantagens do trabalho em grupo e individuais. In CAMPION. M.R., Hidroterapia princípios e práticas $1^{a}$ edição, p.250; 253- São Paulo: Manole, 2000. 